\title{
Numerical analysis of effect of filtration on removal of inclusions in AA3104 aluminum alloy
}

\author{
Xiaoxiong Luo ${ }^{1,2, a}$, Haitao Zhang ${ }^{1, b}$, Dandan Chen ${ }^{3, c}$, Jianzhong Cui ${ }^{1, d^{\star}}$ and \\ Hiromi Nagaumi, ${ }^{3, e}$ \\ ${ }^{1}$ Key Laboratory of Electromagnetic Processing of Materials, Northeastern University, Shenyang, \\ China \\ ${ }^{2}$ Chinalco Rumin Co. Ltd, Fuzhou, China \\ ${ }^{3}$ Chinalco Research Institute of Science and Technology, Beijing, China \\ aluoxx@126.com, bhaitao_zhang@epm.neu.edu.cn, chendd_ddchen@163.com, \\ dz_cui@epm.neu.edu.cn, ${ }^{\mathrm{e}}$ changhai_bw@sinr.cn
}

Keywords: AA3104 aluminum alloy, inclusion, filtration, numerical simulation

Abstract. Melt quality has a crucial influence on the subsequent processing of casting ingot and the performance of the final product. Filtration is one of the most common and important purification methods to remove inclusions in aluminum liquid during melt processing. In this paper, based on the characteristics of the filter to remove inclusions, a mathematic model is built to describe the movement and the removal of inclusions in the process, and this process is calculated with the commercial software Fluent. It was investigated in detail by using the model that the motion and removal of inclusions have different velocity and shape factor in the filtration process. Results revealed that inclusion particles with small shape factor are easy to be removed. The key point of flow velocity is found, which has critical influence on the effects of inclusion removal. The best removal efficiency can be reached when the flow velocity is at $950 \mathrm{~mm} / \mathrm{min}$.

\section{Introduction}

Inclusions in aluminum alloy liquid can have a crucial influence on the subsequent processing of casting ingot and the performance of the final product, such as mechanical properties, corrosion resistance and appearance quality [1,2]. Before the casting ingot, refining, degassing and filtration are main methods used in the production line to removal inclusions [3, 4]. Filtration using ceramic filter plate is one of the common and effective methods. In the late 1970s, the ceramic foam filter (CFF) made of alumina and chromium oxide was invented in United States. Because of the low cost, good purifying effect and strong adaptability of the CFF filter, it was rapidly promoted in the aluminum industries [5]. Studies have shown that the foam (pores) size determines the removal ability of the filter $[6,7]$. For thick filters with small holes, the filtration effect is better, compared to the thin filters with big pores. But how to optimize the use of the filter to ensure the quality of the melt and improve the production efficiency, study for removal and filtration of inclusions still have an important significance for plants of aluminum industries.

In this paper, based on the characteristics of the ceramic filter to remove inclusions, a mathematic model is built to describe the movement and the removal of inclusions in the process, and this process is calculated with the commercial software Fluent. The motion and removal of inclusions with different velocity and shape factor in the filtration process were investigated in detail by the model. The numerical model is verified experimentally by using of PoDFA $[3,8,9]$ technology to test the content of inclusions in an AA3104 aluminum alloy on the production line.

\section{Methods and boundary conditions}

The model with 30 ppi pore distribution is used in this study. The Tetrakaidecahedral is the basic unit of the filter as shown in Fig. 1. The side lengths of the basic unit are $8.48 \mathrm{~mm} \times 8.48 \mathrm{~mm}$, the diameter of the filtering sphere is $8 \mathrm{~mm}$, and the pore diameter in the basic unit is $3.16 \mathrm{~mm}$. In order to 
simplify the calculation, model unit represents the very small part of the filter, and the middle part is the filter. The model is built on the basic filter unit, and the filter is composed of multiple holes. The total height of model unit is $111 \mathrm{~mm}$, including $51 \mathrm{~mm}$ the thickness of the filter as well as the upper and lower aluminum liquid height are $30 \mathrm{~mm}$, recpectively.

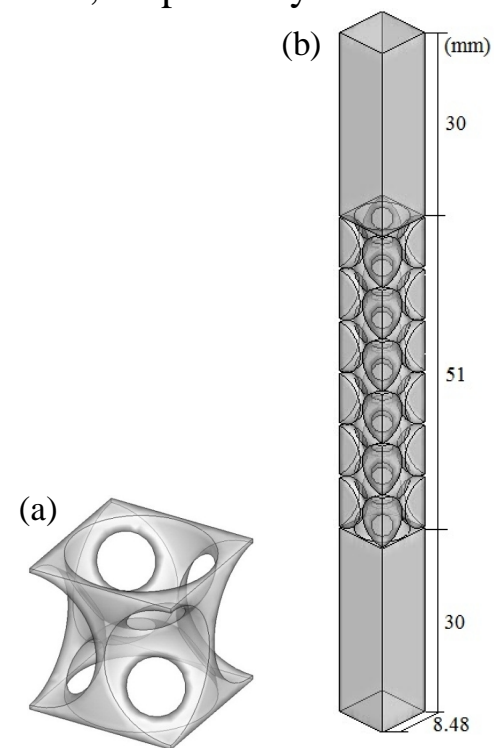

Fig. 1 Basic unit of the mode and basic unit

Fluid flow in the filtering process is set to turbulence model (k-epsilon) with low Reynolds number. In normal conditions, the inclusions in aluminum melt move random, so in Discrete phase modules in the FLUENT software is set as "Discrete Random Walk Model". The boundary conditions in the model are set to periodic boundary conditions. The fluid flows downward depending on the pressure drop. The filter wall in the model is set to "Trap", which means that the particle is absorbed by the wall when they contact with wall of the filter.

In the simulation process, it was considered that the inclusions with different density correspond to different shape factors, and one shape factor corresponds to the five different equivalent diameters. The number of inclusion particles with one equivalent diameter inject into one inlet hole is 286 . The initial time is 1 second, and at every 2 seconds the inclusion particles are inject into the inlet hole, respectively. The total time of simulation run is 400 seconds, and the total number of particles is 1430 . The filtration velocity is set as $250,650,950,650$ and $250 \mathrm{~mm} / \mathrm{min}$, respectively.

\section{Results and discussion}

Velocity field of the aluminum melt. Fig. 2 shows the simulation result under different filtration velocity in three-dimensional velocity field. Because the melt flow is set to the low Reynolds number models with turbulence, the filter has effects of block on the fluid flow. As a result, the fluid flow path is changed, and the fluid flow direction in filter is continual because of effect from the pore wall, so the direction of the velocity adjacent to holes is scattered and disorderly. When the fluid passes through the filter area, the direction of velocity gradually draw closely to the normal direction of the wall. 
(a)

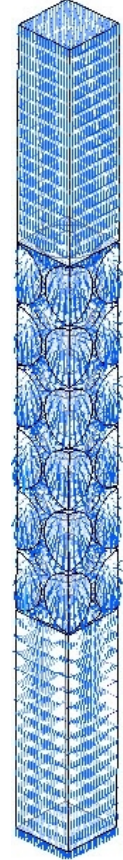

(b)

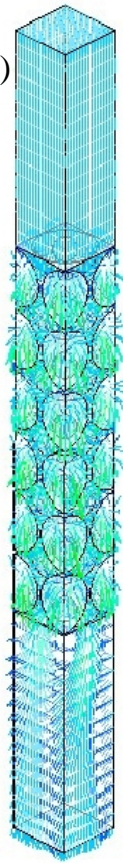

(c)

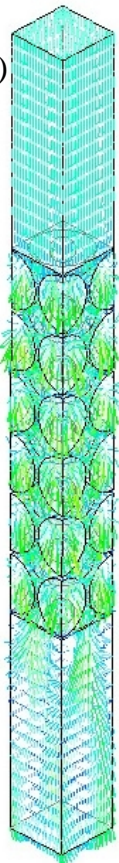

(d)

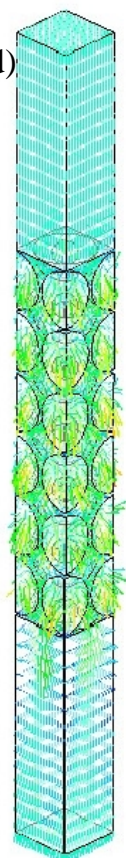

(e)

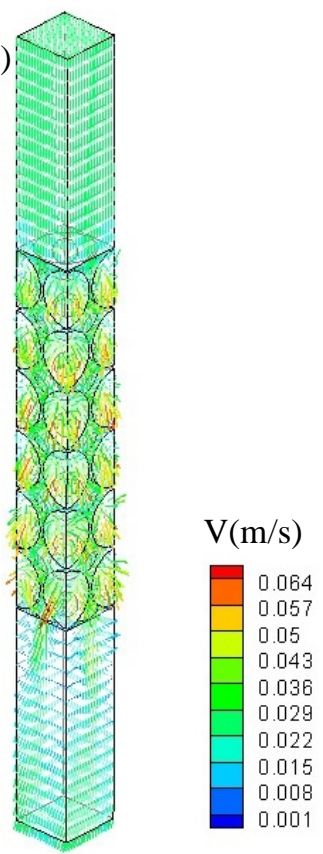

Fig.2 3D-surface plot of vector field under different filtration velocity (a) $250 \mathrm{~mm} / \mathrm{min}$ (b) $650 \mathrm{~mm} / \mathrm{min}$ (c) $950 \mathrm{~mm} / \mathrm{min}$ (d) $1150 \mathrm{~mm} / \mathrm{min}$ (e) $1350 \mathrm{~mm} / \mathrm{min}$

Movement of inclusions. In the filtration rate of $250 \mathrm{~mm} / \mathrm{min}$, the filtration process of $\mathrm{MgO}$ inclusion particles with shape factor of 0.001 at different time points are shown in Fig. 3. Different colors indicate the inclusion particles with different diameters. The particles with minimum diameter of 2 microns are dark blue, and then, the red for particles with the maximum diameter of 50 microns.

(a)

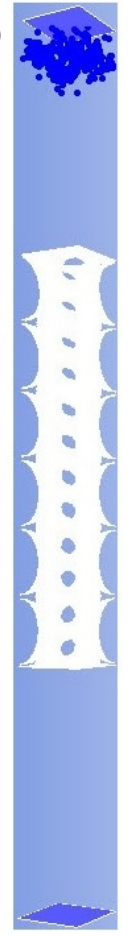

(b)

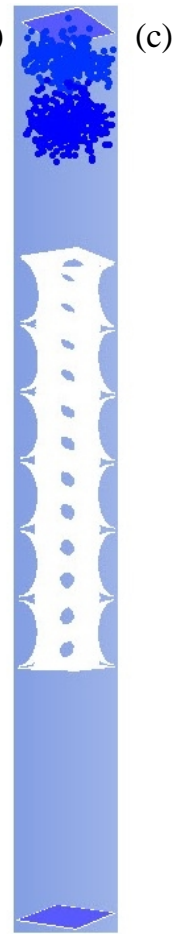

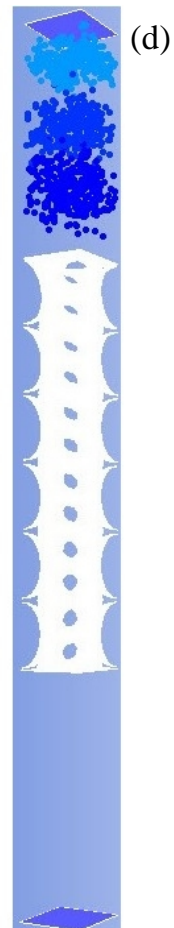
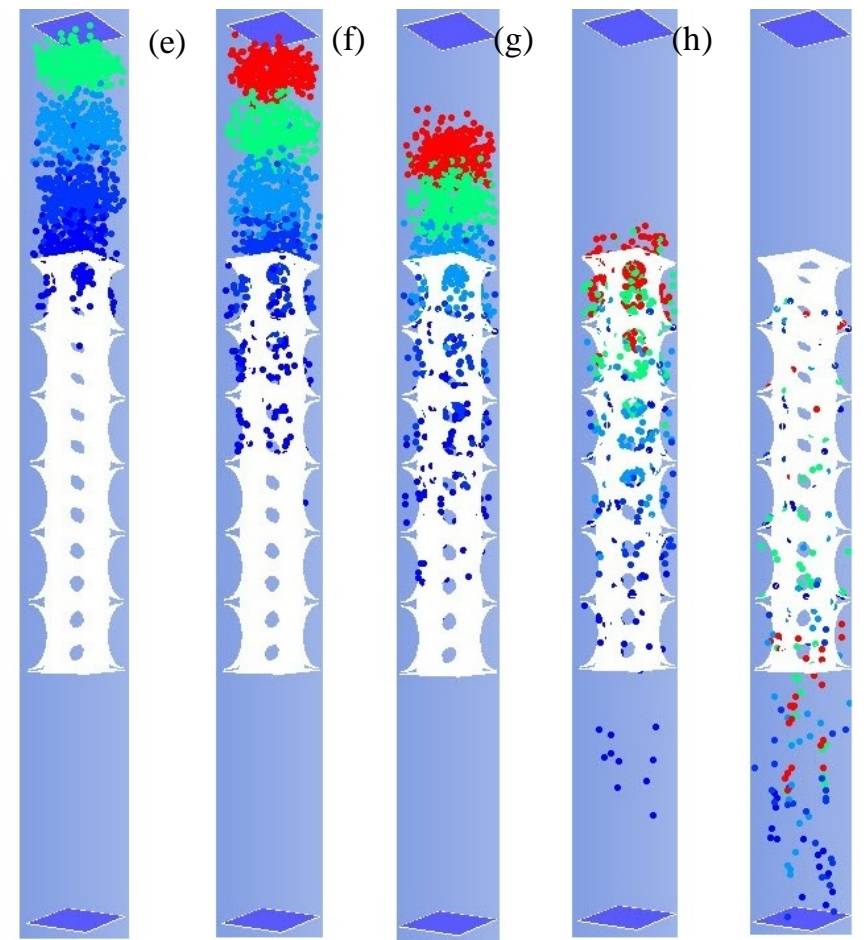

Fig.3 Moving process of inclusions

At the first seconds, particles in the first group with diameter of 2 microns are injected into the model. In the next time points of 3, 5, 7, 9 seconds the particles with diameters of 5, 10, 20 and 50 microns are injected into the model. Five groups of particles are completely in the model, at the same 
time, particles in the first group just come into contact with the filter. In the beginning of the filtration process, most of the particles are removed by filtration, and the number of particles decreases dramatically. Along with melt flows, the movements of particles gradually approach to a steady state. The filter can't remove the entire inclusion particles, so a small number of particles flows through the filter device and remains in the aluminum melt.

Influence of filtration velocity on the filtration. In order to investigate the effect of different filtration velocity on the filtration, $\mathrm{MgO}$ particles is selected in the numerical study. The density of $\mathrm{MgO}$ particles is $3580 \mathrm{~kg} / \mathrm{m}^{3}$, and the shape factor is set as 0.001 . Fig. 4 shows the profiles of filtration percent under different filtration velocity. It is found that in the range of $250 \sim 950 \mathrm{~mm} / \mathrm{min}$, with the increase of filtration velocity, more inclusion particles are filtered out. The best removal effect is found when the flow velocity is at $950 \mathrm{~mm} / \mathrm{min}$. As the filtration speed continues to increase, inclusion removal efficiency decreases.

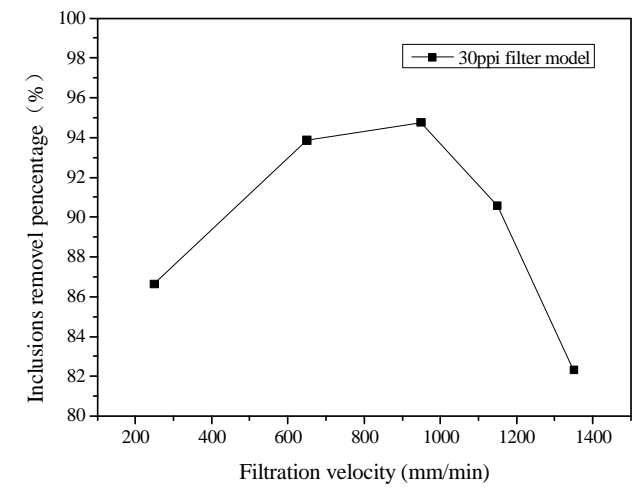

Fig. 4 Percentage of the trapped particles by different filtration velocity

The filtration velocity can influence the turbulent kinetic energy and turbulence dissipation rate in fluid flow. The bigger filtration velocity is, the greater the turbulent kinetic energy and turbulence dissipation rate. When the filtration rate increases from $250 \mathrm{~mm} / \mathrm{min}$ to $650 \mathrm{~mm} / \mathrm{min}$, the turbulent kinetic energy increases. As a result, the movement of inclusion particles accelerates, which means that more particles are captured by the pore wall. When the filtration velocity is beyond the key point of 950 $\mathrm{mm} / \mathrm{min}$, the impact of the fluid and the pore wall is the largest. Consequently, a number of particles captured by the wall will be washed away, and the remove percent of particles decreases.

Influence of shape factor on the filtration. $\mathrm{TiB}_{2}, \mathrm{Al}_{2} \mathrm{O}_{3}, \mathrm{MgAl}_{2} \mathrm{O}_{4}$ and $\mathrm{MgO}$ particles are selected to investigate the influence of density on the remove effect. The densities of the different particles are $4500,3970,3600,3580$ and $3580 \mathrm{~kg} / \mathrm{m}^{3}$, respectively. The shape factors are set as 0.5 and 0.001 , and the filtration speed is $950 \mathrm{~mm} / \mathrm{min}$. The result is shown in Fig. 5 .

It is found that removal percentage of particles with the shape factor of 0.001 is higher than that of particles with the shape factor of 0.5 . The smaller the shape factor value is, the easier the particles are to be captured. It is also found with same volume and same shape factor, particles with bigger density have a more obvious trend to downward, because particles with bigger density have a bigger gravity.

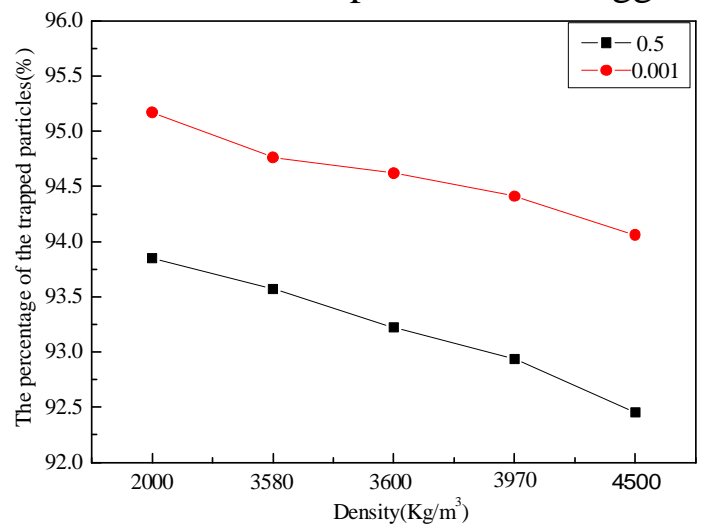

Fig.5 Percentage of the trapped particles of different density of inclusion 


\section{Experimental verification}

Material used in the experiment is AA3104 aluminum alloy, and its nominal component is shown in table 1 . In order to verify the accuracy of the mathematical model, PoDFA method is used in casting production line of some aluminum processing factories to detect the amount of inclusions before and after the filter. The metallographic images of inclusions before and after aluminum melt through $30 \mathrm{ppi}$ filter are shown in Fig. 6. It is found that inclusions in AA3104 aluminum melt are mainly $\mathrm{MgO}$, spinel and $\mathrm{TiB}_{2}$, containing a small amount of Cuboids $(\mathrm{MgO})$ and oxide film. After filtration most of the oxides are removed, and size of inclusions is decreased smaller than that before filtration. The content of inclusions is quantitatively analyzed, as shown in table 2 . After filtration, the content of inclusions is sharply reduced more than $90 \%$. The experimental results agree well with the analysis of numerical simulation.

Table 1 Nominal composition of AA3104 aluminum alloy (wt.\%)

\begin{tabular}{cccccccc}
\hline $\mathrm{Mn}$ & $\mathrm{Mg}$ & $\mathrm{Fe}$ & $\mathrm{Si}$ & $\mathrm{V}$ & $\mathrm{Ti}$ & Others & $\mathrm{Al}$ \\
\hline $0.85 \sim 1.05$ & $1.16 \sim 1.26$ & $0.35 \sim 0.45$ & $0.16 \sim 0.25$ & 0.05 & 0.10 & 0.15 & Balanced \\
\hline
\end{tabular}
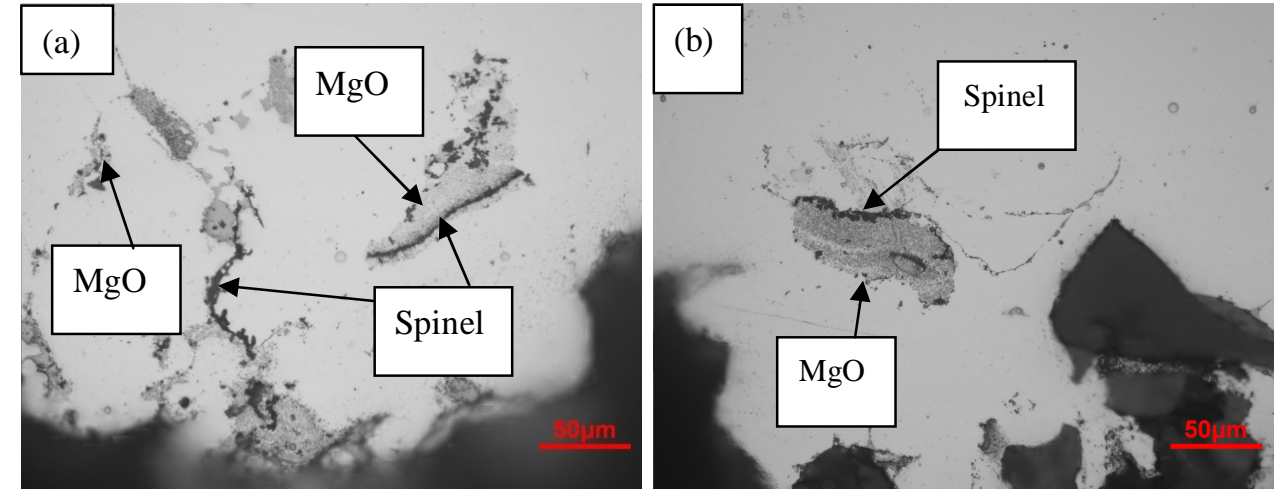

Fig.6 The OM photos of inclusions for samples: (a) before filtration, (b) after filtration

Table 2 Content of inclusions $\left(\mathrm{mm}^{2} / \mathrm{Kg}\right)$

\begin{tabular}{cccccc}
\hline Inclusions & $\mathrm{TiB}_{2}$ & $\begin{array}{c}\text { Spine } \\
1\end{array}$ & $\mathrm{MgO}$ & Cuboids(MgO) & \multirow{2}{*}{ Oxide film } \\
\hline Before filtration & 0.14 & 0.075 & 0.055 & 0.05 & 6 \\
After filtration & 0.011 & 0.004 & 0.003 & 0.0035 & 1 \\
Remove percent $(\%)$ & 92.1 & 93.7 & 94.1 & 93 & 83.3 \\
\hline
\end{tabular}

\section{Conclusions}

The filtering model is established, and the velocity field and movement of inclusions are numerically studied by the FLUENT software. The influences of different filtration velocity and inclusion's shape factors on filtering process are investigated. The main conclusions are as followed:

(1) It is found that the key point of flow velocity which has critical influence on inclusion removal. The best removal effect is reached when the flow velocity is at $950 \mathrm{~mm} / \mathrm{min}$. When the filtration velocity is less than $950 \mathrm{~mm} / \mathrm{min}$, filtering efficiency increases with the filtration velocity increases. When the filtration velocity is greater than $950 \mathrm{~mm} / \mathrm{min}$, the filtration efficiency decreases with the filtration velocity increases.

(2) Inclusion particles with small shape factor are easy to be removed from aluminum liquid.

(3) After filtration, the content of inclusions is sharply reduced more than $90 \%$.

(4) The experimental results agree well with the analysis of numerical simulation.

\section{References}

[1] M. Zhou, D. Shu, K. Li, Mate. Sci. Eng. A, 347(2003) 280-290. 
[2] M. J. Majed, C. Torbjorn, Metall. Mater. Trans. B, 43(2012) 82-91.

[3] X.X. Luo, J.Z. Cui, N. Hiromi, D.D. Chen, Foundary, 63(2014) 138-144.

[4] S. Makarov, D. Apelian, R. Ludwig, AFS Trans., 107(1999) 727-735.

[5] M. Aydin, G. Balik, A.F. Miguel and A.H. Reis, J. Mech. Eng., 51(2005) 495-500.

[6] X. Cao, Mater. Sci. Eng. A, 403(2005) 101-111.

[7] F.A.G. Acosta, A.H. Castillejos, J.M. Alamanza and A. Flores, Metall. Mater.Trans. B, 26(1995) 159-171.

[8] O. Majidi, S.G. Shabestari, M.R. Aboutalebi, J. Mater. Process. Tech., 182(2007) 450-455.

[9] A. Simard, F. Dallaie, J. Proulx, Aluminum Today, 12(2000) 34-36. 